\title{
A density functional study of the effect of hydrogen on electronic properties and band discontinuity at anatase $\mathrm{TiO}_{2} /$ diamond interface
}

Kongping Wu, Meiyong Liao, Liwen Sang, Jiangwei Liu, Masataka Imura, Haitao Ye, and Yasuo Koide

Citation: Journal of Applied Physics 123, 161599 (2018); doi: 10.1063/1.5002176

View online: https://doi.org/10.1063/1.5002176

View Table of Contents: http://aip.scitation.org/toc/jap/123/16

Published by the American Institute of Physics

\section{Articles you may be interested in}

$\mathrm{Al}_{2} \mathrm{O}_{3}$ dielectric layers on $\mathrm{H}$-terminated diamond: Controlling surface conductivity

Journal of Applied Physics 122, 155304 (2017); 10.1063/1.4985808

Deep depletion concept for diamond MOSFET

Applied Physics Letters 111, 173503 (2017); 10.1063/1.4997975

Engineering electric and magnetic dipole coupling in arrays of dielectric nanoparticles Journal of Applied Physics 123, 083101 (2018); 10.1063/1.5018312

Pulse measurement of the hot spot current in a $\mathrm{NbTiN}$ superconducting filament Journal of Applied Physics 123, 083901 (2018); 10.1063/1.4999427

High quality $\mathrm{Al}_{2} \mathrm{O}_{3} /(100)$ oxygen-terminated diamond interface for MOSFETs fabrication Applied Physics Letters 112, 102103 (2018); 10.1063/1.5018403

Assembly of a high-dielectric constant thin $\mathrm{TiO}_{\mathrm{x}}$ layer directly on $\mathrm{H}$-terminated semiconductor diamond Applied Physics Letters 108, 012105 (2016); 10.1063/1.4939650

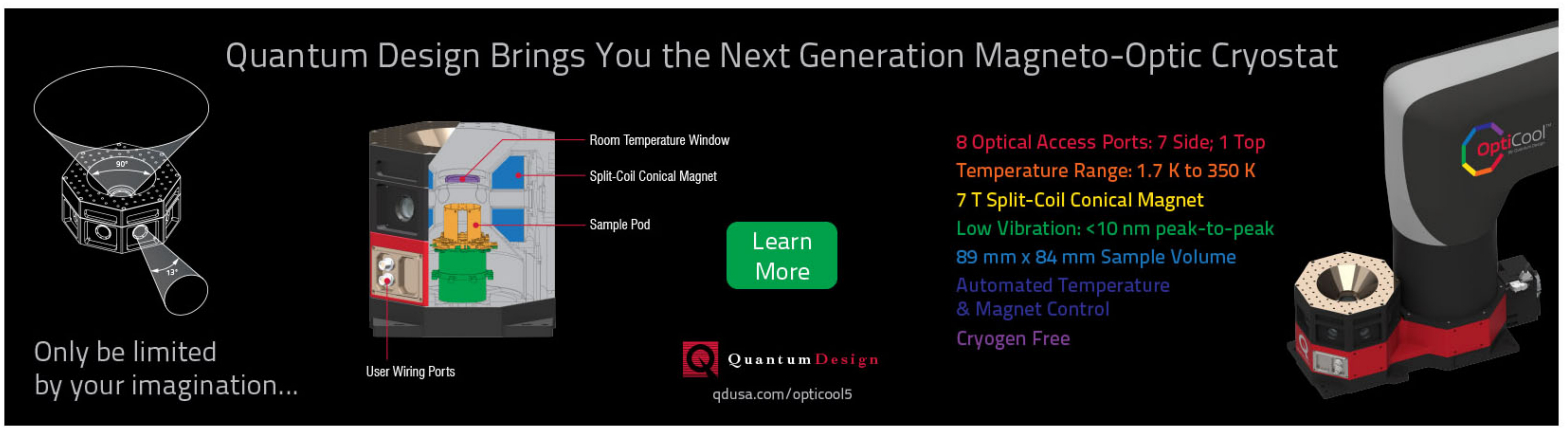




\title{
A density functional study of the effect of hydrogen on electronic properties and band discontinuity at anatase $\mathrm{TiO}_{2} /$ diamond interface
}

\author{
Kongping Wu, ${ }^{1,2, a)}$ Meiyong Liao, ${ }^{1, a)}$ Liwen Sang, ${ }^{3}$ Jiangwei Liu, ${ }^{1}$ Masataka Imura, ${ }^{1}$ \\ Haitao Ye, ${ }^{4}$ and Yasuo Koide ${ }^{5}$ \\ ${ }^{1}$ Research Center for Functional Materials, National Institute for Materials Science (NIMS), Tsukuba, \\ Ibaraki 305-0044, Japan \\ ${ }^{2}$ School of Electrical and Information Engineering, Anhui University of Science and Technology, Huainan, \\ Anhui 232001, China \\ ${ }^{3}$ International Center for Materials Nanoarchitectonics (MANA), National Institute for Materials Science \\ (NIMS), Namiki 1-1, Tsukuba, Ibaraki 305-0044, Japan \\ ${ }_{5}^{4}$ School of Engineering and Applied Science, Aston University, Birmingham B4 7ET, United Kingdom \\ ${ }^{5}$ Research Network and Facility Services Division, National Institute for Materials Science (NIMS), \\ 1-2-1 Sengen, Tsukuba, Ibaraki 305-0047, Japan
}

(Received 30 August 2017; accepted 6 February 2018; published online 22 February 2018)

\begin{abstract}
Tailoring the electronic states of the dielectric oxide/diamond interface is critical to the development of next generation semiconductor devices like high-power high-frequency field-effect transistors. In this work, we investigate the electronic states of the $\mathrm{TiO}_{2} /$ diamond $2 \times 1-(100)$ interface by using first principles total energy calculations. Based on the calculation of the chemical potentials for the $\mathrm{TiO}_{2}$ /diamond interface, it is observed that the hetero-interfaces with the C-OTi configuration or with two $\mathrm{O}$ vacancies are the most energetically favorable structures under the $\mathrm{O}$ rich condition and under Ti-rich condition, respectively. The band structure and density of states of both $\mathrm{TiO}_{2} /$ diamond and $\mathrm{TiO}_{2} / \mathrm{H}$-diamond hetero-structures are calculated. It is revealed that there are considerable interface states at the interface of the anatase $\mathrm{TiO}_{2} /$ diamond hetero-structure. By introducing $\mathrm{H}$ on the diamond surface, the interface states are significantly suppressed. A type-II alignment band structure is disclosed at the interface of the $\mathrm{TiO}_{2} /$ diamond hetero-structure. The valence band offset increases from 0.6 to $1.7 \mathrm{eV}$ when $\mathrm{H}$ is introduced at the $\mathrm{TiO}_{2} /$ diamond interface. Published by AIP Publishing. https://doi.org/10.1063/1.5002176
\end{abstract}

\section{INTRODUCTION}

Diamond is an extreme semiconductor material with a number of outstanding physical, mechanical, and chemical properties, such as a wide bandgap of $5.5 \mathrm{eV}$, high carrier mobility, high breakdown field, maximum hardness, and superior corrosion resistance, which has potential applications in electronics, aerospace, military, and other fields. ${ }^{1-5}$ Recently, significant progress on the growth of high-quality single crystal diamond thin film and semiconductor devices has been achieved. ${ }^{6,7}$ The practical application of semiconductor diamond electronic devices relies on the reduction of the electrical resistivity of diamond by doping impurities and the control of the interface formed between diamond and other materials. Despite the lack of shallow dopants at this moment, the hydrogenated diamond (H-diamond) exhibits a unique p-type conductivity with a hole density of around $10^{13} \mathrm{~cm}^{-2}$ after exposure to air or other surface adsorbates. ${ }^{8}$ Such a conductivity has opened the avenue for the development of metal-semiconductor or metal-oxide-semiconductor field-effect transistors (MOSFETs), which showed exciting performance of high cut-off frequency and capability operating at elevated temperatures. ${ }^{9,10}$ Especially, diamond MOSFETs have been attracting growing interest because of the higher power handling capability. ${ }^{11}$ For example, a high drain current density above $1.3 \mathrm{~A} / \mathrm{mm}$ has been reported. ${ }^{12}$

\footnotetext{
a) Authors to whom correspondence should be addressed: kongpingwu@

126.com and Meiyong.Liao@nims.go.jp
}

The device performance of diamond MOSFETs strongly depends on the gate dielectric materials and oxide/diamond interfaces. Up to now, various oxides such as $\mathrm{Al}_{2} \mathrm{O}_{3}, \mathrm{HfO}_{2}$, and $\mathrm{ZrO}_{2}$ were utilized as the gate dielectric for $\mathrm{H}$-diamond MOSFETs. ${ }^{9-13}$ Titanium oxide $\left(\mathrm{TiO}_{2}\right)$ was also reported by our group as the gate dielectric on H-diamond, resulting in normally off MOSFETs when using a low-temperature oxidation method. ${ }^{14}$ Ideal $\mathrm{TiO}_{2}$ has a high dielectric constant up to 100 , which has the potentiality to control high carrier densities even at small electric fields to improve the output of diamond MOSFETs. ${ }^{15,16}$ In order to fully understand the performance of diamond electronics, theoretical investigation of the oxide/diamond is in demand.

In this work, we perform first-principles calculations on the effect of hydrogen on the electronic properties and band alignments of the anatase $\mathrm{TiO}_{2} / \mathrm{H}$-diamond interface. The calculation on the local density of states indicates that a large number of interface states exist in the band gap. It is disclosed that the anatase $\mathrm{TiO}_{2}$ /diamond interface exhibits a staggered (type II) band alignment. In particular, the introduction of hydrogen leads to an increase in the valence band offset from 0.6 to $1.7 \mathrm{eV}$ at the anatase $\mathrm{TiO}_{2} / \mathrm{H}$-diamond interface.

\section{COMPUTATIONAL METHOD}

The experimental lattice constant of diamond is assumed to be $\mathrm{a}_{0}=3.567 \AA$. The in-plane surface lattice vectors are $\mathrm{n}_{1}[011] \mathrm{a}_{0} / 2$ and $\mathrm{m}_{1}[011] \mathrm{a}_{0} / 2$. For the anatase $\mathrm{TiO}_{2}$, the 
experimental lattice constant of $\mathrm{a}_{1}=3.784 \AA$ is used. ${ }^{17}$ The in-plane surface lattice vectors are $\mathrm{n}_{2}[100] \mathrm{a}_{1}$ and $\mathrm{m}_{2}[010] \mathrm{a}_{1}$, with $\mathrm{n}_{1}, \mathrm{~m}_{1}, \mathrm{n}_{2}$, and $\mathrm{m}_{2}$ being integers.

We utilize the following model to passivate the surface $\mathrm{O}$ atoms of $\mathrm{TiO}_{2}$. In the anatase $\mathrm{TiO}_{2}$, an $\mathrm{O}$ atom with six valence electrons was surrounded by three $\mathrm{Ti}$ atoms and the most stable state for the $\mathrm{O}$ atom has eight electrons to form a closed shell. Therefore, $(8-6) / 3=2 / 3$ pseudo-hydrogens $\left({ }^{2 / 3} \mathrm{H}\right)$ are required to passivate the $\mathrm{O}$ dangling bonds. In addition, hydrogen is used to passivate the back surface $\mathrm{C}$ atoms of the diamond crystal.

The diamond (100) slab model is composed of $8 \mathrm{C}$ atomic layers with $16 \mathrm{C}$ atoms per layer, and the $\mathrm{TiO}_{2}(001)$ slabs have least $8 \mathrm{O}$ atomic layers with $8 \mathrm{O}$ atoms per layer and $4 \mathrm{Ti}$ atomic layers with $4 \mathrm{Ti}$ atoms per layer. These slabs are thick enough to recover the bulk behavior far from the interface. The bottom layers of $\mathrm{C}$ atoms are passivated with hydrogen atoms. The $\mathrm{TiO}_{2}(001)$ surface is terminated by $\mathrm{O}$ with two pseudo-hydrogen atoms with a vacuum space of $15 \AA$ to avoid the interaction between periodic images. All slabs are terminated by the same $\mathrm{O}$ with two pseudohydrogen atoms. Meanwhile, various interface structures are investigated by changing the interface atomic arrangements or compositions. The surface atomic relaxations have little effect on the interface atomic structure.

The total-energy and physical property calculations were carried out with the Vienna ab initio Simulation Package 5.3.3 (VASP) based on the density-functional theory (DFT) within the generalized gradient approximation (GGA). ${ }^{18-20}$ To overcome the shortcomings of the DFT (concerning localized orbitals), the LDA \pm Hubbard $\mathrm{U}$ $(\mathrm{LDA} \pm \mathrm{U}$ ) method is adopted by using the on-site Coulomb interaction instead of the averaged Coulomb energy for localized d electrons, where the value of parameter $\mathrm{U}$ for the Ti $3 d$ orbits is taken as $9.0 \mathrm{eV}^{21}$ The elucidation of the eigenvalue problem and the order of states at the topmost valence band from GGA and GGA $\pm \mathrm{U}$ calculations are discussed in Ref. 21 and will not be repeated here. The Vanderbilt ultrasoft pseudo-potentials and the exchange correlation functional of Perdew, Burke, and Ernzerhof (PBE) for the GGA are used in this simulation. ${ }^{22}$

In the energy minimization, we constrain the lattice constant of the $\mathrm{TiO}_{2} /$ diamond hetero-structure to the average value of those of $\mathrm{TiO}_{2}$ and diamond. The lattice misfit is around $\sim 3 \%$. The three bottom layers of $\mathrm{C}$ atoms and the layer of $\mathrm{H}$ atoms are fixed, whereas the others move freely, in order to facilitate the simulation of a thick diamond substrate and eliminate any strain or stress in the lattice structure as much as possible. The relaxation of the atomic structures is carried out by calculating the Hellmann-Feynman forces until the force on each relaxed atom is less than $0.1 \mathrm{eV} / \AA$. After geometry optimization, the interfacial distance is determined by the minimum energy of the slab. ${ }^{23}$ All calculations are based on pseudopotentials and a plane-wave basis set. The plane wave basis set is included and truncated with a cut-off energy of $500 \mathrm{eV}$. The Monkhorst-Pack scheme is used for the Brillouin zone integration with a $5 \times 9 \times 1 \mathrm{k}$-point mesh, ${ }^{24}$ resulting in the convergence of the electronic total energy within around $10^{-6} \mathrm{eV} / \mathrm{cell}$. The orbital-projected densities of states $(\mathrm{PDOS})$ are calculated with $7 \times 13 \times 1 \mathrm{k}$-point meshes and Gaussian smearing. The calculated interface properties show good convergence with respect to the supercell size [for example, valence-band offset (VBO) variations smaller than $0.1 \mathrm{eV}$ have been found as a function of the number of $\mathrm{C}$ atomic planes]. Due to the asymmetric slab with a net surface dipole density, the periodic boundary conditions imposed on the electrostatic potential give rise to an artificial electric field across the slab. According to Bengtsson's work, ${ }^{25}$ a dipole correction is applied to correct the spurious effect caused by the periodic boundary conditions. At last, the relative interface formation energy is utilized to judge the relative stability of interfaces.

The relative interface formation energy $\Delta \mathrm{E}_{\mathrm{f}}$ can be written as follows: ${ }^{26-28}$

$$
\Delta \mathrm{E}_{\mathrm{f}} \mathrm{A}=\mathrm{E}_{\text {slab }}-\mathrm{E}_{\mathrm{ref}} \pm \mathrm{n}_{\mathrm{Ti}} \mu_{\mathrm{Ti}} \pm \mathrm{n}_{\mathrm{O}} \mu_{\mathrm{O}} \pm \mathrm{n}_{\mathrm{C}} \mu_{\mathrm{C}},
$$

where $E_{\text {slab }}$ is the total energy of the slabs and $E_{\text {ref }}$ is the energy of the reference slab. $\mu_{\mathrm{Ti}}, \mu_{\mathrm{O}}$, and $\mu_{\mathrm{C}}$ are the chemical potentials of $\mathrm{Ti}, \mathrm{O}$, and $\mathrm{C}$, respectively. $\pm \mathrm{n}_{\mathrm{i}}$ is the decrease (increase) in quantity of the ith atom at the interface. A is the interface unit cell area.

According to thermodynamic equilibrium, chemical potentials $\mu_{\mathrm{Ti}}, \mu_{\mathrm{O}}$, and $\mu_{\mathrm{C}}$ in $\mathrm{TiO}_{2}$ and diamond can be written as follows:

$$
\begin{gathered}
\mu^{\text {bulk }}\left(\mathrm{TiO}_{2)}=\mu_{\mathrm{Ti}}+2 \mu_{\mathrm{O}},\right. \\
\mu_{\mathrm{C}}^{\text {bulk }}(\text { diamond })=\mu_{\mathrm{C}} .
\end{gathered}
$$

The heat of formation $\left(\Delta \mathrm{H}_{\mathrm{f}}{ }^{0}\right)$ is defined as

$$
\mu^{\text {bulk }}\left(\mathrm{TiO}_{2}\right)=\mu_{\mathrm{Ti}}+2 \mu_{\mathrm{O}}+\Delta \mathrm{H}_{\mathrm{f}}^{0} .
$$

The variations of $\mu_{\mathrm{Ti}}$ and $\mu_{\mathrm{O}}$ at the interface were limited to the corresponding bulk chemical potentials $\mu_{\mathrm{Ti}}{ }^{\text {bulk }}$ and $\mu_{\mathrm{O}}{ }^{0}$, respectively. The boundary conditions are written as

$$
\Delta \mathrm{H}_{\mathrm{f}}^{0} / 2 \leq \mu_{\mathrm{O}}-\mu_{\mathrm{O}}^{0} \leq 0 .
$$

The chemical potential $\mu_{\mathrm{Ti}}{ }^{\text {bulk }}$ is the total energy per atom of the metallic Ti and $\mu_{\mathrm{O}}{ }^{0}$ is taken to be half of the energy of an $\mathrm{O}_{2}$ molecule. Similarly, $\mu_{\mathrm{C}}^{\text {bulk }}$ is the total energy per atom of the diamond and $\mu_{\mathrm{H}}{ }^{0}$ is half of the energy of an $\mathrm{H}_{2}$ molecule. The relationship between the relative interface formation energy and $\mu_{\mathrm{O}}-\mu_{\mathrm{O}}{ }^{0}$ is expressed as

$$
\begin{aligned}
\Delta \mathrm{E}_{\mathrm{f}} \mathrm{A}= & \mathrm{E}_{\text {slab }}-\mathrm{E}_{\mathrm{ref}} \pm \mathrm{n}_{\mathrm{Ti}} \mu^{\text {bulk }}\left(\mathrm{TiO}_{2}\right) \pm \mathrm{n}_{\mathrm{H}} \mu_{\mathrm{H}}^{0} \\
& \pm\left(2 \mu_{\mathrm{Ti}}-\mathrm{n}_{\mathrm{O}}\right) \mu_{\mathrm{O}} \pm \mathrm{n}_{\mathrm{C}} \mu_{\mathrm{C}}^{\text {bulk }} \text { (diamond). }
\end{aligned}
$$

Note that the calculated value of $\Delta \mathrm{H}_{\mathrm{f}}{ }^{0}$ is $-10.15 \mathrm{eV}$, which is in good agreement with the value $(-9.6 \pm 0.8 \mathrm{eV})$ reported by Knauth. ${ }^{29}$

\section{RESULTS AND DISCUSSION}

\section{A. The relative interface formation energy}

There are two types of interfaces for the $\mathrm{TiO}_{2} /$ diamond hetero-structure: one is made of oxygen atoms (C-OTi) and 

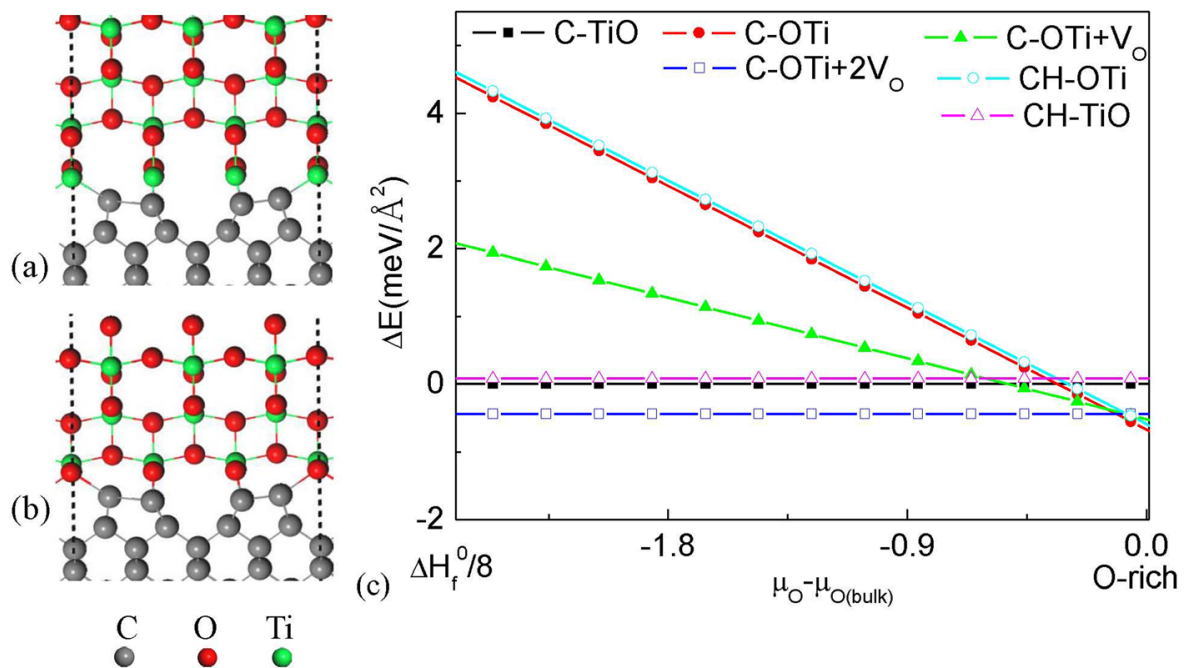

FIG. 1. The Slab models of $\mathrm{TiO}_{2} /$ diamond hetero-interfaces with the $\mathrm{C}$ - $\mathrm{TiO}$ configuration (a) and C-OTi configuration (b), and (c) the calculated functional relationship between the relative interface formation energy and $\mu_{\mathrm{O}}-\mu_{\mathrm{O}}{ }^{0}$. the other is made of $\mathrm{Ti}$ atoms $(\mathrm{C}-\mathrm{TiO})$ for the first layer of $\mathrm{TiO}_{2}$ at the interface. For the diamond, particular test calculations about the unreconstructed diamond surface and the reconstructed $2 \times 1-(100)$ surface have been carried out. The interface formation energy for the unreconstructed diamond surface is higher than that of the reconstructed diamond surface at the interface of the $\mathrm{TiO}_{2} /$ diamond hetero-structure. In addition, the interface with $\mathrm{C}$-TiO has the highest interface formation energy. In addition, the geometries of the $2 \times 1-(100)$ and $2 \times 1-(100): \mathrm{H}$ surface reconstructions are the most stable surfaces for the (100) diamond, ${ }^{30-33}$ which are utilized as the initial condition. Here, the hydrogenterminated diamond $2 \times 1-(100)$ surface is also investigated to understand the effect of hydrogen on the electronic properties of the hetero-interface.

For the C-OTi interface, the $\mathrm{O}$ vacancy $\left(\mathrm{C}-\mathrm{OTi}+\mathrm{V}_{\mathrm{O}}\right)$ is also considered, which is common in interfacial point defects. ${ }^{34}$ To discuss the thermodynamic stability of these hetero-interfaces, the relative interface formation energy is calculated, as shown Fig. 1(c). The energy of the slab with the $\mathrm{C}$-TiO interface is chosen as the reference, which is set to be zero. It is observed that the C-OTi hetero-interface with two $\mathrm{O}$ vacancies $\left(\mathrm{C}-\mathrm{OTi}+2 \mathrm{~V}_{\mathrm{O}}\right)$ and the heterointerface with the C-OTi configuration are the most energetically favorable structures under the Ti-rich condition and under the O-rich condition, respectively. In addition, it is revealed that the introduction of $\mathrm{H}$ to some extent weakens the stability of the interface. Here, the C-OTi interface is first calculated. $\mathrm{H}$ is then introduced at the interface to examine its effect on the electronic properties of the C-OTi interface.

\section{B. Band structure and density of states}

As is well known, a high density of interface states will lead to poor device performance. To develop an engineering solution to reduce the interface states, it is necessary to elucidate the origin of the interface states. The band structures (left panel of each figure in Fig. 2) are plotted along high symmetry k-points $\mathrm{G}(0,0,0)-\mathrm{F}(0.5,0,0)-\mathrm{Q}(0.5,0.5,0)-\mathrm{G}$ $(0,0,0)-Z(0,0.5,0)$. The total densities of states (TDOSs) of anatase $\mathrm{TiO}_{2} /$ diamond and anatase $\mathrm{TiO}_{2} / \mathrm{H}$-diamond heterostructures are calculated and shown in the right panel of each figure in Fig. 2. The (blue) dotted region in the band structure represents the contribution of the "bulk" diamond band structure (note that the computed bulk band gap is $4.20 \mathrm{eV}$ ). The black lines indicate the projected band structure for the interface model.
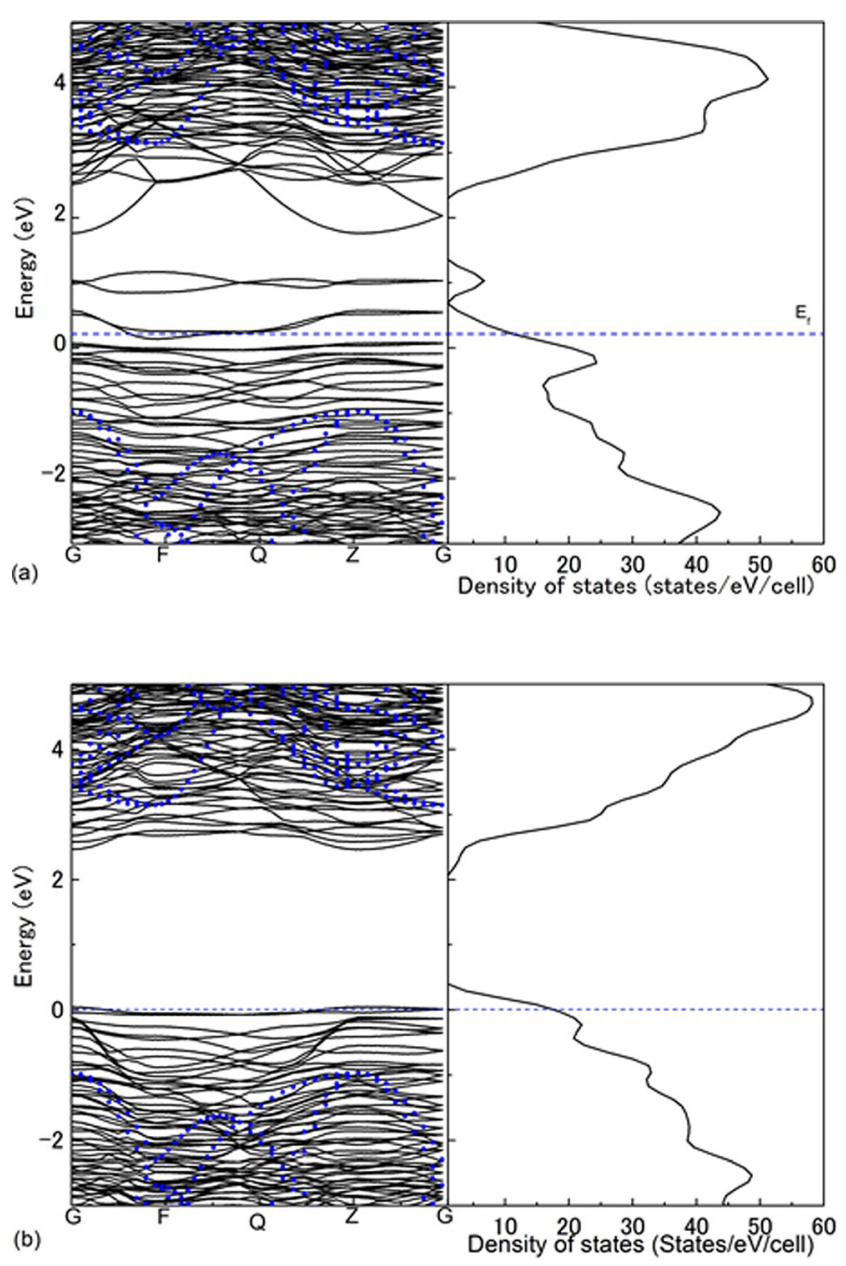

FIG. 2. Band structures (left panel) and total density of states (right panel) for interfaces (a) C-OTi and (b) CH-OTi, respectively. The (blue) dotted region represents the diamond bulk band structure. The dashed horizontal line indicates the Fermi energy. 
In the case of the C-OTi interface [Fig. 2(a)], the bulk diamond gap region (from -0.98 to $3.22 \mathrm{eV}$ ) is filled with multiple gap states. The DOS shows a large density of gap states, which results in the Fermi level pinning. While for the CH-OTi interface, little gap states are observed, as shown in Fig. 2(b) of the band structures and DOSs. The interface gap states can be significantly reduced at the $\mathrm{TiO}_{2} / \mathrm{H}$-diamond interface due to the introduction of $\mathrm{H}$.

The formation of interface states and the change of the density of the interface states near the Fermi energy are further investigated by the layer-projected density of states (LDOSs) for the $\mathrm{TiO}_{2} /$ diamond and $\mathrm{TiO}_{2} / \mathrm{H}$-diamond interface, as shown in Fig. 3. The LDOSs of the hetero-structure are divided into three parts: the bulk $\mathrm{TiO}_{2}$ region, the bulk diamond region, and the interface region. The bulk $\mathrm{TiO}_{2}$ region and the bulk diamond region are far from the interface almost not being affected by the interface.

The main changes in the LDOSs relative to the bulk occur at the $\mathrm{C}$ layer and $\mathrm{TiO}$ bilayer adjacent to the interface. For the C-OTi interface, the most contribution to the density of the interface states near the Fermi energy is from the first and second layer of C (1L-C and $2 \mathrm{~L}-\mathrm{C}) 2 s 2 p$ orbitals, $3 \mathrm{~L}-\mathrm{C}$, and $4 \mathrm{~L}-\mathrm{C} 2 p$ orbitals. According to the TiO's LDOSs, there is little contribution to the density of the interface states near the Fermi energy from the $\mathrm{O} 2 p$ orbital and the Ti $3 \mathrm{~d}$ orbital in the $1 \mathrm{~L}-\mathrm{TiO}$ layer. In comparison, the LDOSs of $1 \mathrm{~L}-\mathrm{C}$ and $2 \mathrm{~L}-\mathrm{C} 2 s 2 p$ orbitals greatly decrease at the $\mathrm{CH}-\mathrm{OTi}$ interface. Therefore, the introduction of $\mathrm{H}$ at the $\mathrm{TiO}_{2} /$ diamond heterostructure strongly suppresses the interface states. In addition, from the viewpoint of the energy position of LDOSs, the introduction of $\mathrm{H}$ pushes the Fermi level bending upward about $0.7 \mathrm{eV}$ for the $\mathrm{TiO}_{2} / \mathrm{H}$-diamond hetero-structure. This is consistent with the negative electron affinity observed in the H-diamond surface. ${ }^{35,36}$

\section{Charge transfer}

To clarify the bonding mechanism and to investigate the transfer of electrons between the adjacent layers at the interface of the $\mathrm{TiO}_{2} /$ diamond and the $\mathrm{TiO}_{2} / \mathrm{H}$-diamond hetero-structures, plane-averaged electron density difference $\Delta \rho(\mathrm{z})$ along the direction perpendicular to the interface is calculated. First, the electron density difference $\Delta \rho(\mathrm{x}, \mathrm{y}, \mathrm{z})$ is calculated according to the following relationship: ${ }^{37,38}$

$$
\begin{aligned}
\Delta \rho(\mathrm{x}, \mathrm{y}, \mathrm{z})= & \rho\left(\mathrm{TiO}_{2}(001) / \text { diamond }\right) \\
& -\left(\rho(\text { diamond })+\rho\left(\mathrm{TiO}_{2}(001)\right)\right),
\end{aligned}
$$

where $\rho\left(\mathrm{TiO}_{2}(001) /\right.$ diamond $)$ is the electron density of the $\mathrm{TiO}_{2}(001) /$ diamond hetero-structure, and $\rho$ (diamond) and $\rho\left(\mathrm{TiO}_{2}(001)\right)$ are the electron densities of the reconstructed diamond $2 \times 1-(100)$ surface and $\mathrm{TiO}_{2}(001)$ surface, respectively, with exactly the same geometries as those of the $\mathrm{TiO}_{2}(001) /$ diamond hetero-structure.

The calculated results of charge density difference of the interfaces of the $\mathrm{TiO}_{2} /$ diamond and the $\mathrm{TiO}_{2} / \mathrm{H}$-diamond hetero-structures are shown in Fig. 4 in the form of contour plots. Figure 4(a) shows the character of charge transfer for the $\mathrm{TiO}_{2} / \mathrm{H}$-diamond hetero-structure. It is seen that charges are depleted in the region of $\mathrm{C}$ plane and $\mathrm{Ti}$ plane. Meanwhile, charges are accumulated in the region of $\mathrm{O}$ plane near the interface, which indicates the atomic interaction and the formation of new chemical bonds to stabilize the interface. On the other hand, for the $\mathrm{TiO}_{2} / \mathrm{H}$-diamond interface, much less charge transfer occurs between $\mathrm{TiO}_{2}$ and $\mathrm{H}$-diamond at the interface, which results in less interface states. The charges transfer only takes place between $\mathrm{C}$, $\mathrm{H}, \mathrm{O}$, and $\mathrm{Ti}$ atoms near the interface, which suggests a localized character.

To further analyze the charge transfer quantitatively, the plane-averaged (x,y) charge density difference $\Delta \rho(\mathrm{z})$ for interfaces of the $\mathrm{TiO}_{2} /$ diamond and the $\mathrm{TiO}_{2} / \mathrm{H}$-diamond heterostructures is calculated, as displayed in Figs. 4(c) and 4(d), respectively. The amount of charge transfer (q) is calculated by integrating $\Delta \rho(\mathrm{z})$ over the full $\mathrm{z}$ range. The plane-averaged $(\mathrm{x}, \mathrm{y})$ charge density difference for the hetero-structure all clearly deviates from zero near the interface, suggesting a
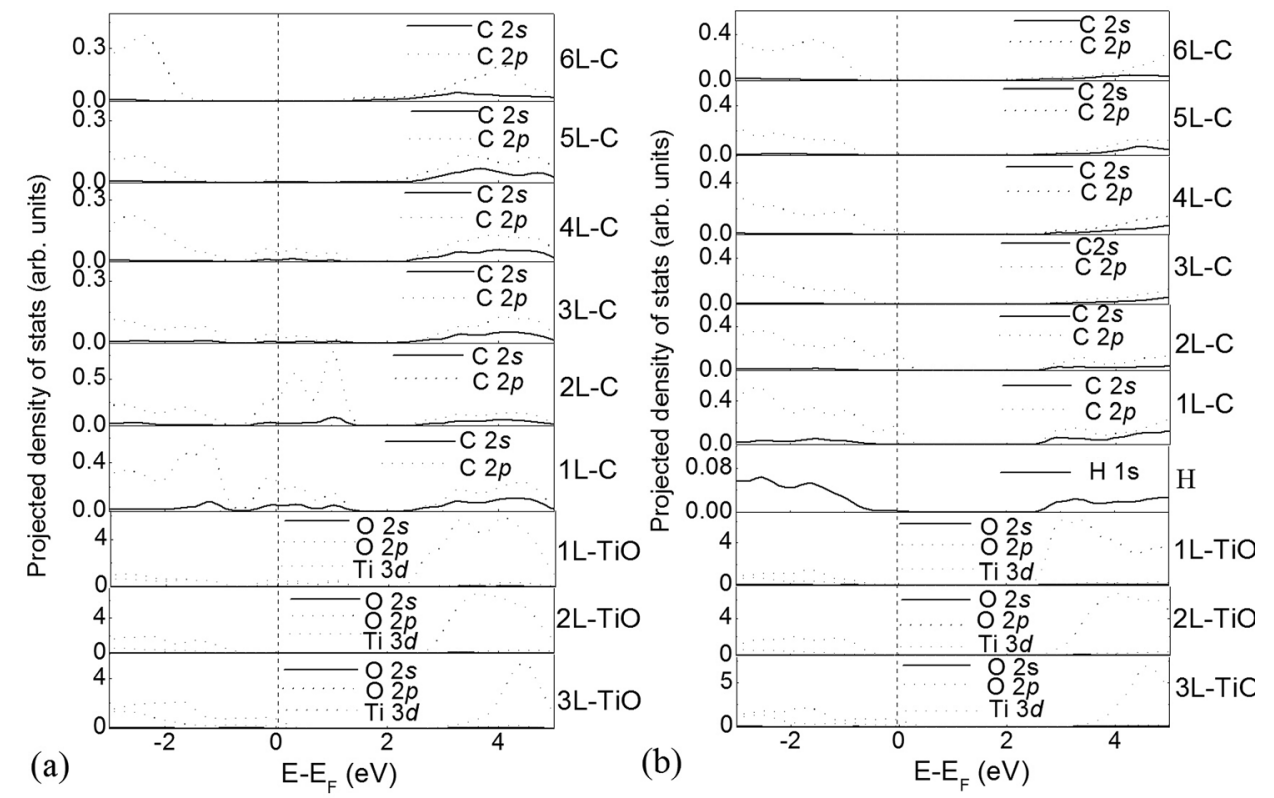

FIG. 3. The PDOS for (a) the $\mathrm{TiO}_{2} /$ diamond hetero-structure and (b) the $\mathrm{TiO}_{2} / \mathrm{H}$-diamond hetero-structure. The layer number is counted from the interface to the surface. The dashed vertical line indicates the Fermi energy. 
(a)

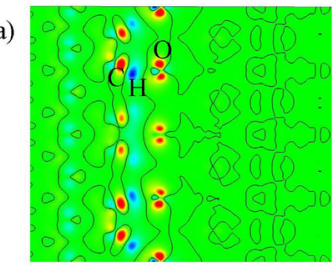

(b)

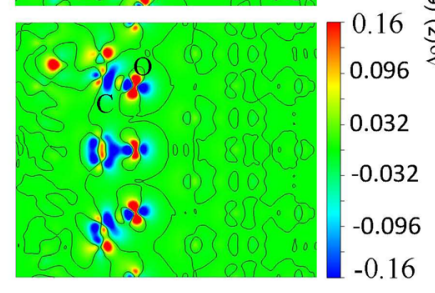

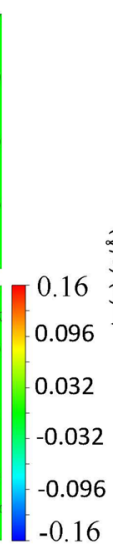

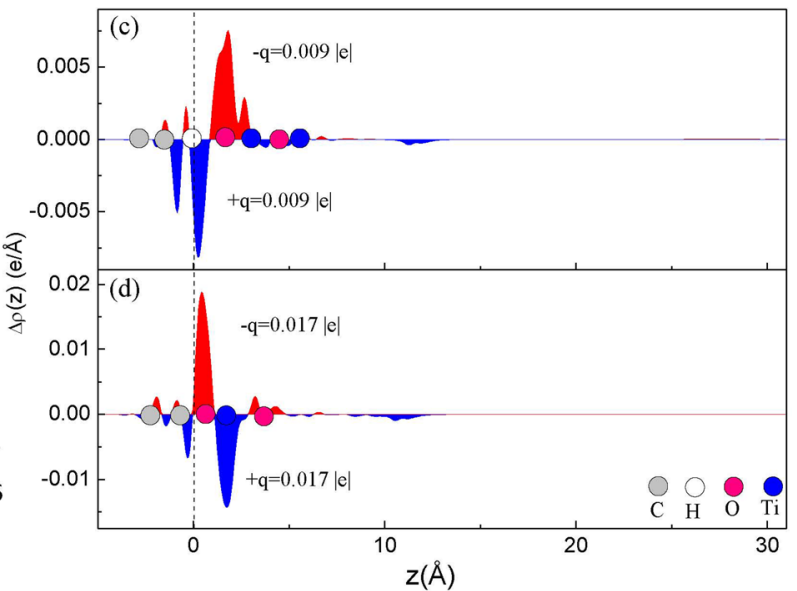

FIG. 4. Contour plots of the valence electronic-charge density difference for the interface of (a) $\mathrm{TiO}_{2}(001) / \mathrm{H}$-diamond and (b) $\mathrm{TiO}_{2}(001) /$ diamond. The atoms that intersect the contour plane are labeled. (Blue: charges are depleted, Red: charges are accumulated, and the unit is $0.001 \mathrm{e} / \AA^{3}$ ) The plane-averaged electron density difference, $\Delta \rho(\mathrm{z})$, for the systems (c) $\mathrm{TiO}_{2}(001) / \mathrm{H}$-diamond and (d) $\mathrm{TiO}_{2}(001) /$ diamond. The positions of the atoms are indicated by solid circles, and $\mathrm{q}$ is the charge transfer calculated by integrating $\Delta \rho(\mathrm{z})$ over the full $\mathrm{z}$ range. considerable number of charge transfer between the diamond and $\mathrm{TiO}_{2}(001)$ slabs. As shown in Figs. 4(c) and 4(d), for the interfaces of $\mathrm{TiO}_{2}(001) / \mathrm{H}$-diamond and $\mathrm{TiO}_{2}(001) /$ diamond hetero-structures, respectively, charge transfer decreases from 0.17 to 0.09 due to the introduction of $\mathrm{H}$ in the $\mathrm{TiO}_{2}(001) /$ $\mathrm{H}$-diamond hetero-structure. Therefore, the introduction of $\mathrm{H}$ plays a role to suppress the charge transfer between $\mathrm{TiO}_{2}(001)$ and the diamond.

\section{Band offset calculations}

To characterize the hetero-structure interface, we calculate the energy band alignment or band offset of the interfaces. The energy band alignment at the interface is evaluated by the well established "bulk plus lineup" method, ${ }^{39}$ in which the macroscopic averaged electrostatic potentials are used as the reference energies as proposed by de Valle and Martin. ${ }^{40}$ According to the derivation of the macroscopic averaged electrostatic potentials for a bulk semiconductor, the band edges are matched up at the interface to determine the band lineups. The band gap is usually underestimated by the DFT method, which results in the too-low-lying conduction band. Therefore, we focus on the alignment of the valence-band edge $\left(\mathrm{E}_{\mathrm{V}}\right)$ and Fermi level $\left(\mathrm{E}_{\mathrm{F}}\right)$. The experimental values of $3.2 \mathrm{eV}$ (Ref. 41) and $5.5 \mathrm{eV}$ (Ref. 42) for the band gaps of $\mathrm{TiO}_{2}$ and diamond, respectively, are used to determine the conduction-band edge.

The planar average of the electrostatic potential is calculated for each hetero-structure. The oscillating function with dips in Fig. 5 corresponds to the atomic planes. The macroscopic average electrostatic potentials along the $\mathrm{z}$ direction as a function of $\mathrm{z}$ are also displayed in Fig. 5. In addition, the conduction band minimum $\left(\mathrm{E}_{\mathrm{C}}\right)$ and valence band maximum $\left(\mathrm{E}_{\mathrm{V}}\right)$ are marked in Fig. 5. The average valence-band offset (VBO) of the C-OTi interface and the $\mathrm{CH}-\mathrm{OTi}$ interface together with the corresponding conduction-band offsets (CBOs) are revealed in Fig. 5.

Figure 5(b) discloses that the VBO increases from 0.6 to $1.7 \mathrm{eV}$ due to the introduction of $\mathrm{H}$. Correspondingly, the $\Delta \mathrm{E}_{\mathrm{C}}$ increases from 2.9 to $4.0 \mathrm{eV}$. The overall band alignment is a type II (staggered) band alignment for the anatase $\mathrm{TiO}_{2} /$ diamond. On the other hand, by gradually decreasing the interfacial O content from $100 \%$ to $50 \%$ at the C-OTi interface $\left(\mathrm{C}-\mathrm{OTi} \pm \mathrm{V}_{\mathrm{O}}\right.$ and $\left.\mathrm{C}-\mathrm{OTi} \pm 2 \mathrm{~V}_{\mathrm{O}}\right)$, the valence band offset increases from $0.6 \mathrm{eV}$ for the C-OTi interface, $0.8 \mathrm{eV}$ for the $\mathrm{C}-\mathrm{OTi} \pm \mathrm{V}_{\mathrm{O}}$ interface, to $0.9 \mathrm{eV}$ for the $\mathrm{C}-\mathrm{OTi} \pm 2 \mathrm{~V}_{\mathrm{O}}$ interface. Experimentally, the VBO was estimated to be around $2.6 \mathrm{eV}$ for the TiOx/diamond junction by XPS, ${ }^{14}$ which is higher that of the present DFT calculation for the
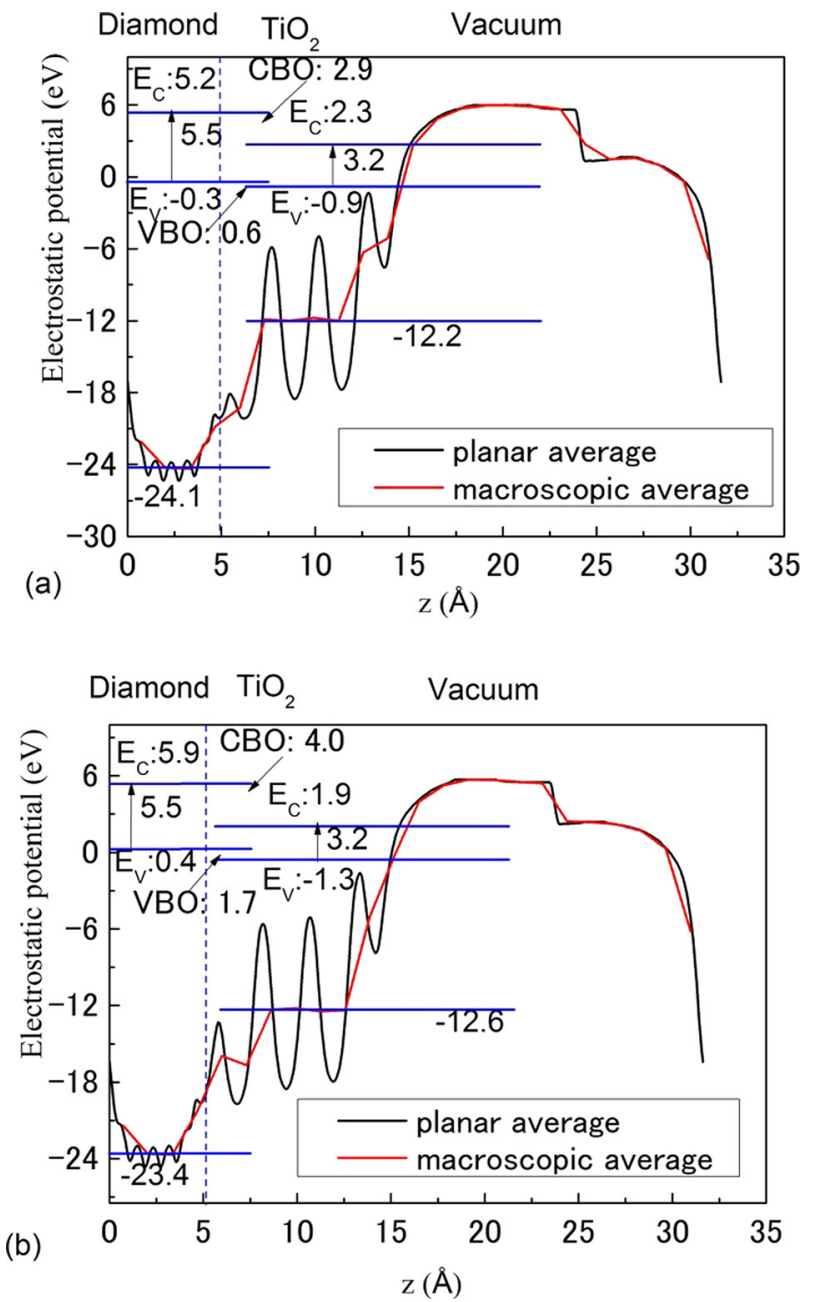

FIG. 5. (a) Planar and macroscopic average of the electrostatic potential as a function of the position along the direction perpendicular to the interface, and the calculated band alignment and average valence-band offset for (a) $\mathrm{TiO}_{2}(001) /$ diamond and (b) $\mathrm{TiO}_{2}(001) / \mathrm{H}$-diamond. The interfaces are marked by the dashed vertical line. The band edges are determined separately from the calculations on bulk diamond and $\mathrm{TiO}_{2}$. 


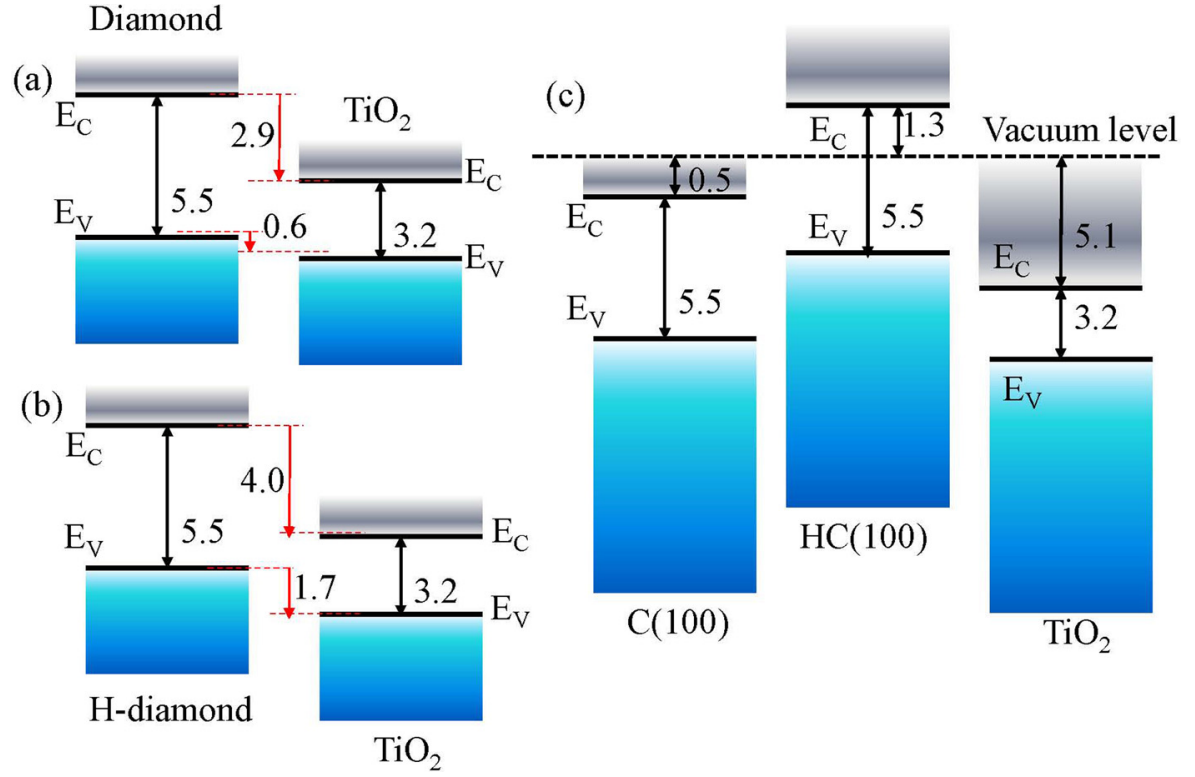

FIG. 6. Schematic representation of the relative position of the valence band and conduction-band edges of (a) $\mathrm{TiO}_{2}(001) /$ diamond and (b) $\mathrm{TiO}_{2}(001) /$ $\mathrm{H}$-diamond as obtained from the "bulk plus lineup" method. (c) Energy band profile for the $\mathrm{TiO}_{2}(001) / \mathrm{C}(100)$ heterostructures without and with $\mathrm{H}$ according to the experimental values of electron affinity.
$\mathrm{TiO}_{2} /$ diamond. The difference is possibly due to the presence of a large number of defects at the interface of the TiOx/diamond junction.

Based on the above calculated VBOs, the relative positions of the valence-band and conduction-band edges of the $\mathrm{TiO}_{2}(001) /$ diamond and $\mathrm{TiO}_{2}(001) / \mathrm{H}$-diamond heterostructures obtained from the "bulk plus lineup" method are schematically displayed in Figs. 6(a) and 6(b), respectively. According to experimental values of the electron affinity of $5.1 \mathrm{eV}$ for $\mathrm{TiO}_{2}{ }^{43} 0.5 \mathrm{eV}$ positive electron affinity for the diamond (100) surface without $\mathrm{H}$ termination, and a $1.3 \mathrm{eV}$ negative electron affinity for the H-diamond (100) surface, ${ }^{44}$ the energy band profiles for the $\mathrm{TiO}_{2}(001) / \mathrm{C}(100)$ heterostructures without and with $\mathrm{H}$ are presented in Fig. 6(c). The $\mathrm{VBO}$ and $\mathrm{CBO}$ are estimated ranging from 2.3 to $4.1 \mathrm{eV}$ and from 4.6 to $6.4 \mathrm{eV}$, respectively. In our calculation, the minimum and maximum $\mathrm{VBO}$ values are 0.6 and $1.7 \mathrm{eV}$, and the minimum and maximum $\mathrm{CBO}$ values are 2.9 and $4.0 \mathrm{eV}$, respectively.

Our results reveal that the band offsets are rather sensitive to the details of the interface. The band alignments for the $\mathrm{TiO}_{2}(001) /$ diamond and $\mathrm{TiO}_{2}(001) / \mathrm{H}$-diamond heterostructures show a typical electronic character of the type-II staggered band configurations, which is desirable to confine the hole between the diamond and $\mathrm{TiO}_{2}$. These merits indicate that $\mathrm{TiO}_{2}$ can be used as a high-k gate insulator on the $\mathrm{H}$-diamond surface for the fabrication of MOSFETs. However, there is still interface states at the interface of anatase the $\mathrm{TiO}_{2} / \mathrm{H}$-diamond hetero-structure despite that $\mathrm{H}$ greatly decreases the interface states. These states may affect the device performance based on the $\mathrm{TiO}_{2} /$ diamond heterostructure. The surface passivation of diamond, i.e., other thin oxides, by combining with the interfacial hydrogen content control can be used to further suppress the interface states for MOSFET fabrication based on the $\mathrm{TiO}_{2} / \mathrm{H}$-diamond (100). The $k$ value of 100 is much higher than those insulators reported on diamond, ${ }^{9-13}$ which offers the advantage of low gate voltage control of the channel charges of diamond MOSFETs.

\section{SUMMARY}

To summarize, the first principles method was utilized to investigate the impact of $\mathrm{H}$ on the electronic properties of $\mathrm{TiO}_{2} /$ diamond hetero-interfaces. It was observed that the relative interface formation energy with specific interfacial oxygen content changes with respect to the oxygen chemical potential. Under an oxygen-rich condition, the C-OTi interface is the most stable interface. By analyzing the band structures and DOSs, it is concluded that there are considerable interface states at the interface of the anatase $\mathrm{TiO}_{2} /$ diamond hetero-structure. These interface states mainly result from the $\mathrm{C} 2 p$ orbit. The introduction of $\mathrm{H}$ strongly suppresses the interface states to a certain extent and increases band offsets simultaneously. The type-II alignment hetero-structure with relatively large CBO and VBO is observed, which is in agreement with the experimental findings. We note that although the simulation is not the realistic amorphous $\mathrm{TiO}_{\mathrm{x}}$ on diamond, the findings could be a guideline for the fabrication of diamond electronic devices.

\section{ACKNOWLEDGMENTS}

This work was partially supported by the scholarship from China Scholarship Council (CSC) under the Grant No. 201508340047, the Anhui province university Outstanding Talent Cultivation Program (gxfxZD2016077), China Postdoctoral Science Foundation (2016M601993), and JSPS KAKENHI (Grant Number 15H03999).

${ }^{1}$ D. Liu, H. R. Sun, J. W. Pomeroy, D. Francis, F. Faili, D. J. Twitchen, and M. Kuball, Appl. Phys. Lett. 107, 251902 (2015).

${ }^{2}$ E. Monroy, F. Omnès, and F. Calle, Semicond. Sci. Technol. 18, R33 (2003).

${ }^{3}$ M. Y. Liao, L. W. Sang, T. Teraji, M. Imura, J. Alvarez, and Y. Koide, Jpn. J. Appl. Phys., Part 1 51, 090115 (2012).

${ }^{4}$ M. Schmitt, D. Paulmier, and T. L. Huu, Thin Solid Films 343-344, 226 (1999).

${ }^{5}$ A. BenMoussa, A. Soltani, U. Schühle, K. Haenen, Y. M. Chong, W. J. Zhang, R. Dahal, J. Y. Lin, H. X. Jiang, H. A. Barkad, B. BenMoussa, D. Bolsee, C. Hermans, U. Kroth, C. Laubis, V. Mortet, J. C. De Jaeger, B. Giordanengo, M. Richter, F. Scholze, and J. F. Hochedez, Diamond Relat. Mater. 18, 860 (2009). 
${ }^{6}$ J. Isberg, J. Hammersberg, E. Johansson, T. Wikstrom, D. J. Twitchen, A J. Whitehead, S. E. Coe, and G. A. Scarsbrook, Science 297, 1670 (2002).

${ }^{7}$ M. Kasu, K. Ueda, Y. Yamauchi, A. Tallaire, and T. Makimoto, Diamond Relat. Mater. 16, 1010 (2007).

${ }^{8}$ C. E. Nebel, C. Sauerer, F. Ertl, M. Stutzmann, C. F. O. Graeff, P. Bergonzo, O. A. Williams, and R. Jackman, Appl. Phys. Lett. 79, 4541 (2001).

${ }^{9}$ H. Kawarada, H. Tsuboi, T. Naruo, T. Yamada, D. Xu, A. Daicho, T. Saito, and A. Hiraiwa, Appl. Phys. Lett. 105, 013510 (2014).

${ }^{10} \mathrm{~K}$. Takahashi, M. Imamura, and K. Hirama, Appl. Phys. Lett. 104, 072101 (2014).

${ }^{11}$ M. Imura, R. Hayakawa, E. Watanabe, M. Y. Liao, Y. Koide, and H. Amano, Phys. Status Solidi RRL 5, 125 (2011).

${ }^{12}$ K. Hirama, H. Sato, Y. Harada, H. Yamamoto, and M. Kasu, Jpn. J. Appl. Phys. 51, 090112 (2012).

${ }^{13}$ J. W. Liu, M. Y. Liao, M. Imura, A. Tanaka, H. Iwai, and Y. Koide, Sci. Rep. 4, 6395 (2014).

${ }^{14}$ J. Zhao, J. W. Liu, L. W. Sang, M. Y. Liao, D. Coathup, M. Imura, B. G. Shi, C. Z. Gu, Y. Koide, and H. T. Ye, Appl. Phys. Lett. 108, 012105 (2016).

${ }^{15}$ D. Wei, T. Hossain, N. Y. Garces, N. Nepal, H. M. Meyer III, M. J. Kirkham, C. R. Eddy, and J. H. Edgar, ECS J. Solid State Sci. Technol. 2, N110 (2013).

${ }^{16}$ S. W. Wright, C. P. Judge, M. J. Lee, D. F. Bowers, F. Derek, M. Dunbar, C. D. Wilson, and D. Craig, J. Vac. Sci. Technol. B 30, 062202 (2012).

${ }^{17}$ A. Idris and Z. Majidnia, Appl. Mech. Mater. 606, 99 (2014).

${ }^{18}$ K. P. Wu, J. D. Ye, K. Tang, J. Qi, S. M. Zhu, and S. L. Gu, Comput. Mater. Sci. 109, 225 (2015).

${ }^{19}$ G. Kresse and J. Furthmüller, Comput. Mater. Sci. 6, 15 (1996).

${ }^{20} \mathrm{P}$. Hohenberg and W. Kohn, Phys. Rev. 136, B864 (1964); W. Kohn and L. J. Sham, ibid. 140, A1133 (1965).

${ }^{21}$ T. Qi, I. Grinberg, and A. M. Rappe, Phys. Rev. B 83, 224108 (2011).

${ }^{22}$ J. P. Perdew, K. Burke, and M. Ernzerhof, Phys. Rev. Lett. 77, 3865 (1996).

${ }^{23}$ P. T. Xu, Q. Tang, and Z. Zhou, Nanotechnology 24, 305401 (2013).
${ }^{24}$ H. J. Monkhorst and J. D. Pack, Phys. Rev. B 13, 5188 (1976).

${ }^{25}$ L. Bengtsson, Phys. Rev. B 59, 12301 (1999).

${ }^{26}$ Z. Y. Li, J. L. Yang, J. G. Hou, and Q. S. Zhu, Phys. Rev. B 65, 100507 (2002).

${ }^{27}$ O. Romanyuk, T. Hannappel, and F. Grosse, Phys. Rev. B 88, 115312 (2013).

${ }^{28}$ O. Romanyuk, O. Supplie, T. Susi, M. M. May, and T. Hannappel, Phys. Rev. B 94, 155309 (2016).

${ }^{29}$ P. Knauth and H. L. Tuller, J. Appl. Phys. 85, 897 (1999).

${ }^{30}$ H. Seo, A. B. Posadas, C. Mitra, A. V. Kvit, J. Ramdani, and A. A. Demkov, Phys. Rev. B 86, 075301 (2012).

${ }^{31}$ J. van der Weide, Z. Zhang, P. K. Baumann, M. G. Wensell, J. Bernholc, and R. J. Nemanich, Phys. Rev. B 50, 5803 (1994).

${ }^{32}$ J. Furthmüller, J. Hafner, and G. Kresse, Phys. Rev. B 53, 7334 (1996).

${ }^{33}$ S. J. Sque, R. Jones, and P. R. Briddon, Phys. Rev. B 73, 085313 (2006).

${ }^{34}$ M. M. Hassan and K. Larsson, J. Phys. Chem. C 118, 22995 (2014).

${ }^{35}$ J. Ristein, W. Stein, and L. Ley, Phys. Rev. Lett. 78, 1803 (1997).

${ }^{36}$ A. K. Tiwari, J. P. Goss, P. R. Briddon, A. B. Horsfall, N. G. Wright, R. Jones, and M. J. Rayson, Europhys. Lett. 108, 46005 (2014).

${ }^{37}$ E. Flage-Larsen, O. M. Løvvik, C. M. Fang, and G. Kresse, Phys. Rev. B 88, 165310 (2013).

${ }^{38}$ S. Gudmundsdóttir, W. J. Tang, G. Henkelman, H. Jónsson, and E. Skúlason, J. Chem. Phys. 137, 164705 (2012).

${ }^{39}$ K. P. Wu, J. Qi, B. Peng, K. Tang, J. D. Ye, S. M. Zhu, and S. L. Gu, Acta Phys. Sin. 64, 0187304 (2015).

${ }^{40}$ C. G. V. de Valle and R. M. Martin, Phys. Rev. B 35, 8154 (1987).

${ }^{41}$ X. Chen and S. S. Mao, Chem. Rev. 107, 2891 (2007).

${ }^{42}$ C. E. Nebel, Nat. Mater. 2, 431 (2003).

${ }^{43}$ D. O. Scanlon, C. W. Dunnill, J. Buckeridge, S. A. Shevlin, A. J. Logsdail, S. M. Woodley, C. R. A. Catlow, M. J. Powell, R. G. Palgrave, I. P. Parkin, G. W. Watson, T. W. Keal, P. Sherwood, A. Walsh, and A. A. Sokol, Nat. Mater. 12, 798 (2013).

${ }^{44}$ F. Maier, J. Ristein, and L. Ley, Phys. Rev. B 64, 165411 (2001). 